\title{
Schlafapnoe-Prädiktoren für Mann und Frau
}

\author{
Bei den Prädiktoren für Schlafapnoe gibt es offenbar Geschlechtsunterschiede. Besonders hinschauen \\ sollte man bei dicken, schnarchenden Männer und bei hypertonen, postmenopausalen Frauen.
}

Forscher werteten die Akten von 272.705 Patienten aus schlafmedizinischen Zentren in den USA aus, bei denen eine schlafbezogene Atmungsstörung vermutet wurde. Die Daten umfassten Alter, Body Mass Index (BMI) und Halsumfang, mehrere selbstberichtete Symptome einer obstruktiven Schlafapnoe (OSA) sowie etliche Komorbiditäten. Zusätzlich lagen die Auswertungen einer nächtlichen Polygrafie vor, die den nasalen Atemgasfluss, die transkutane Sauerstoffsättigung, die Herzfrequenz, das Schnarchgräusch und die Kopfbewegungen aufzeichnete.

Der stärkste Prädiktor für eine OSA - definiert über einen Wert ab 5 im
Apnoe-Hypopnoe-Index - war ein Alter $>45$ Jahre. Allerdings lag die Odds Ratio (OR) älterer Frauen verglichen mit jüngeren Frauen bei 5,0, während der Vergleich bei Männern nur eine OR von 3,4 ergab.

Männer gaben das Symptom Schnarchen häufiger an als Frauen $(65,9 \%$ vs. $56,3 \%, \mathrm{p}<0,001)$, der prädiktive Wert war aber gleich hoch. Beobachtete Atemstillstände und ein BMI $\geq 30 \mathrm{~kg} / \mathrm{m}^{2}$ waren bei Männern bessere Prädiktoren einer OSA als bei Frauen. Dagegen hatte die anamnestische Erwähnung des Vorliegens einer arteriellen Hypertonie bei Frauen einen besseren prädiktiven Wert als bei Männern $(\mathrm{p}<0,001)$.
- Cairns S, Poulos G, Bogan R. Sex differences in sleep apnea predictors and outcomes from home sleep apnea testing. Nat Sci Sleep. 2016;8:197-205

\section{KOMMENTAR}

Die wichtigste Aussage ist, dass ein höheres Alter bei Frauen deutlich klarer als bei Männern ein sehr wichtiger Prädiktator für eine OSA ist, was sicher an postmenopausalen Veränderungen liegt. Interessant ist aber auch, dass Schnarchen geschlechtsunabhängig einen relevanten prädiktiven Wert hat. Die für Frauen häufig erwähnten OSA-typischen Angaben wie Insomnie, Depression und Gebrauch von Schlafmitteln hatten dagegen keinen prädiktiven Wert.

Prof. Dr. med. K. Rasche

\section{Eine Oberarmarterie wie eine Perlenschnur}

Eine 68-jährige Frau kam mit Brustschmerzen und Belastungsdyspnoe in die Nothilfe. Man vermutete eine instabile Angina pectoris, zumal sie sich fünf Jahre zuvor einer Bypass-Operation unterzogen hatte. Beim Versuch einer Koronarangiografie über die A. radialis ergaben sich Schwierigkeiten, den Führungsdraht und den Katheter über die Ellenbeuge hinaus vorzuführen. Bei einer Angiografie der A. brachialis ergab sich das Bild einer Perlschnur (Abb.), vereinbar mit einer fibromuskulären Dysplasie der Arterie.

Dabei handelt es sich um ein nicht entzündliches und nicht arteriosklerotisches Krankheitsbild. Die fibromuskuläre Dysplasie wird am häufigsten im Bereich der Karotiden und der Nierenarterien beobachtet, kann aber prinzipiell an jeder anderen Arterie auftreten. Viele Patienten haben keine Beschwerden, manche entwickeln aber eine Symptomatik im Sinne einer Durchblutungsstörung im Ausbreitungsgebiet. Je nach Ausbreitungsgebiet können Nierenversagen, Schlaganfall, Bauchschmerzen oder Claudicatio intermittens die Folge sein.

Interessanterweise waren bei dieser Patientin der Radialis-Puls rechts tastbar und sogar der Allen-Test normal. Die Koronarangiografie gelang schließlich über einen Femoralis-Zugang. In dieser Arterie fand sich keine fibromuskuläre Dysplasie, die koronaren Bypass-Gefäße waren offen. Die Brustschmerzen der Patientin bilde-

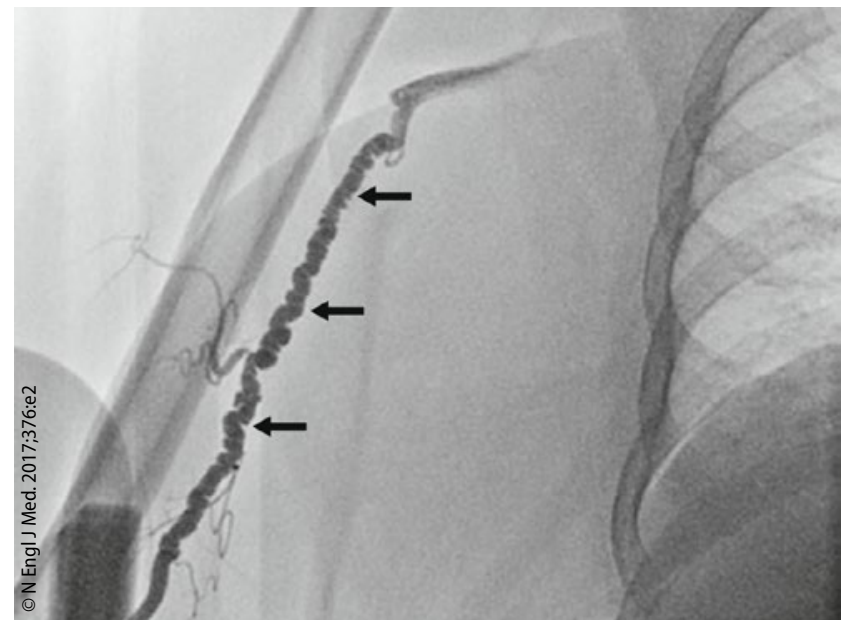

Dysplasie der A. brachialis in der Angiografie.

ten sich zurück und wurden als nicht kardial interpretiert. Am nächsten Tag konnte sie beschwerdefrei entlassen werden

Prof. Dr. med. H. S. FüeßI

- Miller MB, Flores DR III (mistyblue56@ gmail.com): Fibromuscular dysplasia of the brachial artery. NEngl J Med. 2017;376:e2 